\title{
TILLANDSIA MOLLIS VAR. REPTANS (BROMELIACEAE), UNA NOVEDAD DEL noroeste de Argentina
}

\author{
HUGO AYARDE ${ }^{1}$ y EVA BULACIO 2
}

\begin{abstract}
Resumen: Tillandsia mollis H. Hrom. \& W. Till es una rara especie de las montañas del sur de Bolivia. En este artículo se da a conocer T. mollis var. reptans, una entidad que habita el sector alto de las Sierras de Calilegua (Jujuy, Argentina). La nueva variedad difiere de la típica por su menor porte, su hábito reptante y el color castaño-rojizo de sus flores. Se incluye una descripción detallada de la novedad, una clave para diferenciar las dos variedades y se ilustra con dibujos, fotografías y un mapa de distribución.
\end{abstract}

Palabras clave: Argentina, Diaphoranthema, Sierras de Calilegua, Tillandsia.

Summary: Tillandsia mollis var. reptans (Bromeliaceae), a novelty from northwestern Argentina. Tillandsia mollis $\mathrm{H}$. Hrom. \& W. Till is a rare species from the mountains of southern Bolivia. This paper reports $T$. mollis var. reptans, an entity that inhabits the high level of the Sierras of Calilegua (Jujuy, Argentina). The new variety differs from the typical for its smaller size, creeping habit and brown-reddish flowers. A detailed description of the novelty, a key to differentiate the two varieties, drawings, photographs and a map, are included.

Key words: Argentina, Diaphoranthema, Sierras of Calilegua, Tillandsia.

\section{INTRODUCCIÓN}

Las montañas húmedas del noroeste de Argentina y del sur de Bolivia constituyen una gran unidad ecológica, biológica y cultural. Específicamente en lo biológico ello se pone de manifiesto, entre otros aspectos, en las numerosas especies de plantas que comparten y en novedades que constantemente son reportadas a medida que se avanza en su exploración biológica (Bulacio \& Ayarde, 2009). Es el caso de Tillandsia mollis H. Hrom. \& W. Till, una especie que habita por encima del nivel superior de los bosques montanos. Esta especie fue descripta para el sur de Bolivia (Till \& Hromadnik, 1983) y, hasta el presente, sólo era conocida para unas pocas localidades del departamento Tarija.

\footnotetext{
${ }^{1}$ Instituto de Ecología. Fundación Miguel Lillo, Miguel Lillo 251, 4000 San Miguel de Tucumán, Tucumán, Argentina. hayarde@lillo.org.ar

2 Laboratorio de Taxonomía Fanerogámica. Fundación Miguel Lillo, Miguel Lillo 251, 4000 San Miguel de Tucumán, Tucumán, Argentina. ebulacio@lillo.org.ar
}

Tillandsia mollis pertenece al subgénero Diaphoranthema (Beer) Baker, cuyas especies son reunidas en seis grupos -llamados "agregados" (Till, 1992a, Donadío et al., 2015)- y dentro de éstos al agregado Recurvata (Till, 1992b), caracterizado por hojas dísticas de sección más o menos circular, inflorescencias 1 (2) floras y sépalos débilmente fusionados. Tal cual lo señalan los autores de la especie, T. mollis guarda cierta semejanza morfológica con T. usneoides (L.) L. (también incluida en el agregado Recurvata), pero difiere de ésta en hábito y porte y en algunos caracteres florales. En cuanto a lo primero, T. mollis es rupícola, claramente más robusta y, en comparación, su tallo es más corto y con conspicua presencia de raíces, lo que contrasta con el hábito epifítico y con los largos y flexibles tallos y ausencia de raíces que caracteriza a T. usneoides. En cuanto al aspecto floral, difiere por la nerviación e indumento de los sépalos: en $T$. mollis están cubiertos por escamas en su porción dorsal libre y los 3 nervios centrales se unen en el extremo, mientras que en T. usneoides los sépalos son glabros y los nervios centrales son libres pues no llegan al ápice. Dentro de Diaphoranthema 
también puede ser confundida con algunas entidades englobadas en el agregado Capillaris (sensu Till, 1989), más precisamente con la rara entidad del área serrana de Córdoba descripta como $T$. dependens Hieron. ex Mez f. perusneoides (Mez) A. Cast. (Castellanos, 1945) y posteriormente incluida en $T$. capillaris Ruiz \& Pav. f. hieronymi (Mez) L. B. Sm. (Smith \& Downs, 1977), en T. virescens Ruiz \& Pav. (Till, 1989) o en T. capillaris Ruiz \& Pav. (Castello $\&$ Galetto, 2013). De esta entidad (T. dependens f. perusneoides) y de todas las incluidas en el agregado Capillaris, T. mollis difiere fundamentalmente por el grado de fusión de los sépalos (que va desde todos connados en la base a uno libre y los dos restantes fusionados sólo en la base) y por el mayor largo de los entrenudos del tallo.

Si bien T. mollis es reportada en la Web como planta cultivada, en la naturaleza es considerada rara debido a su restringida distribución. Sólo hay documentados tres registros de la especie (H. Hromadnik HR9088, Heger s.n. y Serrano et al. 6076) y todos ellos de las montañas de Tarija, en el sur de Bolivia (Base Tropicos, 2015). La localidad tipo, Abra del Cóndor, se ubica al este de la ciudad de Tarija en la ruta hacia Entre Ríos. Otra localidad, vagamente indicada y erróneamente georreferenciada en la Base Tropicos del MO, probablemente se ubique en el curso superior del Pilaya, río ubicado al norte de Tarija y que constituye parte del límite departamental con Chuquisaca. Un tercer y más reciente registro la reporta para una localidad más al sur, en el área montañosa del noroeste de la Reserva Nacional de Flora y Fauna Tariquía.

Colectas recientes dan cuenta de la presencia en las Sierras de Calilegua (Jujuy, Argentina) de una entidad que por sus caracteres florales responde a T. mollis, pero que difiere claramente de ésta en aspectos vegetativos tales como hábito y porte, razón por la cual la proponemos aquí como una variedad.

\section{Materiales y Métodos}

Para su determinación se emplearon los métodos clásicos en taxonomía. El material estudiado, procedente de las Sierras de Calilegua (Jujuy, Argentina), fue obtenido en numerosas campañas entre 2010 y 2014, realizadas en el marco de un estudio florístico del sector alto de las Sierras. Se consultaron los herbarios CORD, LIL, MCNS, SI, de Argentina; BOLV, HSB, LPB, USZ, de Bolivia y complementariamente se hicieron consultas virtuales de tipos de T. mollis depositados en HAL, NY, W, WU.

\section{Resultados}

Tillandsia mollis H. Hrom. \& W. Till var. reptans Ayarde \& Bulacio, var. nov. TIPO: Argentina. Jujuy, dpto. Valle Grande, Sierras de Calilegua, Quebrada de Cortaderas, 2650 m s.m., 9-IX-2010, Bulacio \& Ayarde 1753 (holotypus LIL, isotypus CORD). Figs. 1, 2.

A varietate typica minorem magnitudinem, habitus coloniae, radicantes brevibus caulibus postratis, erectis brevis paucifollis simplici vel parum ramosis, differt.

Planta reptante, pequeña; tallos postrados radicantes, con uno a tres tallos erguidos (raro más), uno por nudo. Tallos erguidos simples o pauci ramificados, cilíndricos de hasta $5 \mathrm{~cm} \mathrm{x}$ 1,8-2 mm; entrenudos 7-14 (-17) mm de longitud, totalmente cubiertos por la vaina foliar. Hojas dísticas, 3 a 5 por tallo, perpendiculares a éste y con el extremo curvado hacia arriba, densamente cinéreo lepidotas; vaina convoluta poco diferenciada, unida hasta los 2/3, 8-14 x 5-7 mm en la porción libre extendida, glabra en la base luego lepidota y con un delgado margen hialino en la porción libre; lámina cilíndrica, ligeramente atenuada hacia el ápice, 30-70 x 1,1-1,8 mm. Inflorescencia uniflora, terminal o subterminal. Bráctea del escapo menor, igual o excediendo la flor. Bráctea floral oblonga, igual o más corta que los sépalos, 6-9 x 4-5 mm, apiculada, densamente lepidota y con 7 a 9 nervios conspicuos (5 unidos en el ápice). Flor sésil de hasta $10 \mathrm{~mm}$ de longitud, tubo cubierto parcialmente por las brácteas, lóbulos reflexos; sépalos aovados, subiguales, 7-9 x 2-3 mm, 5-nervados (3 unidos en el ápice), con el ápice agudo, lepidotos en la mitad superior; pétalos obovados, $10-11 \times 2 \mathrm{~mm}$, glabros, constrictos; lóbulos con ápice obtuso, castañooscuros; estambres inclusos o levemente exertos, filamentos lineares $5 \mathrm{~mm}$ de longitud; anteras basifijas, amarillas, oblongas, apiculadas, $2 \mathrm{~mm}$ de longitud; ovario ovoide, verde amarillento, 1,5 x 1 
H. Ayarde y E. Bulacio - Tillandsia mollis var. reptans, de Argentina
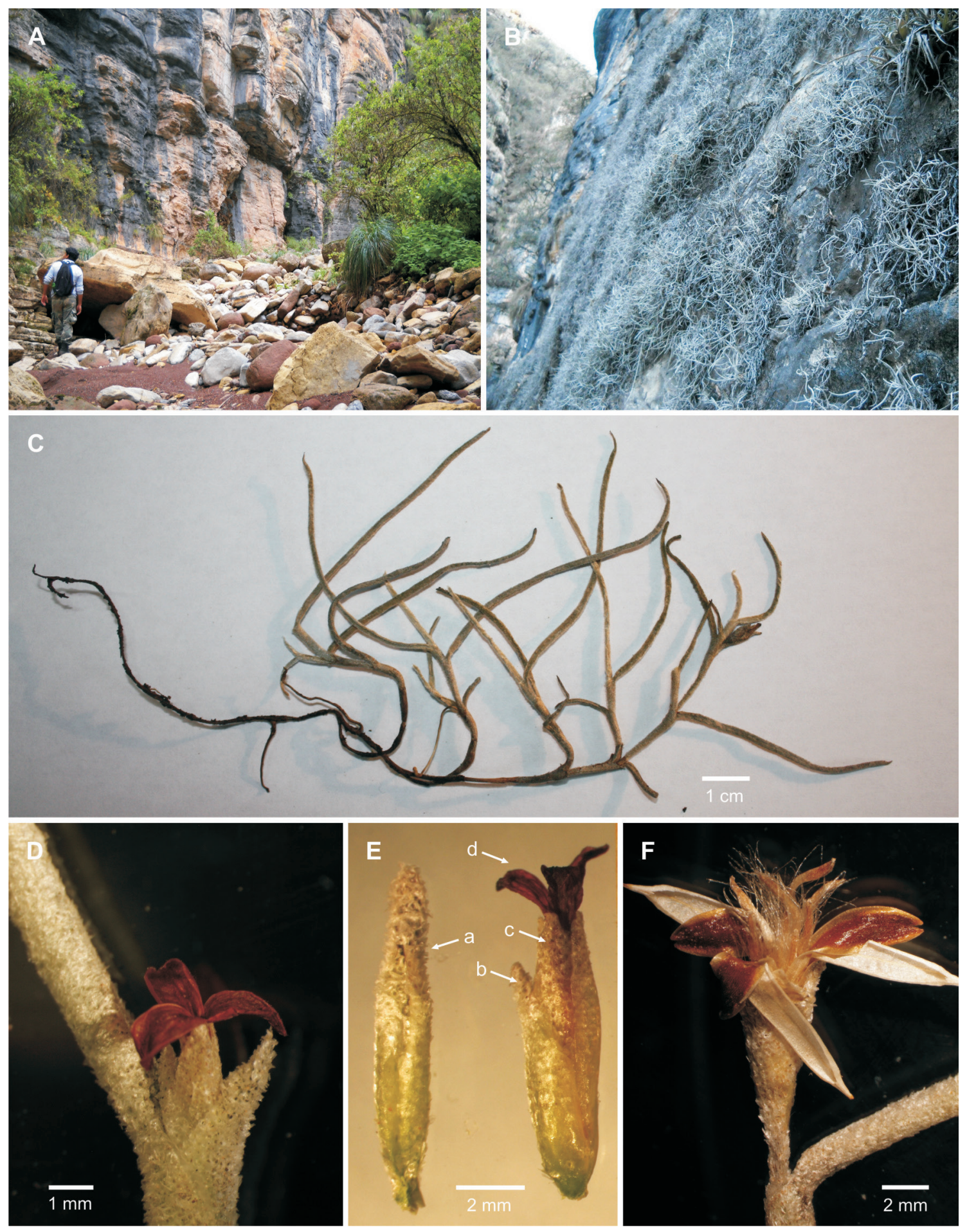

Fig. 1. Tillandsia mollis var. reptans. A: Hábitat, Qda. de Cortaderas, Sierras de Calilegua. B: Hábito de crecimiento. C: Aspecto de la planta. D: Inflorescencia. E: Partes de la inflorescencia, a: bráctea del escapo, b: bráctea floral, c: sépalos, d: pétalos. F: Fruto en dispersión. 

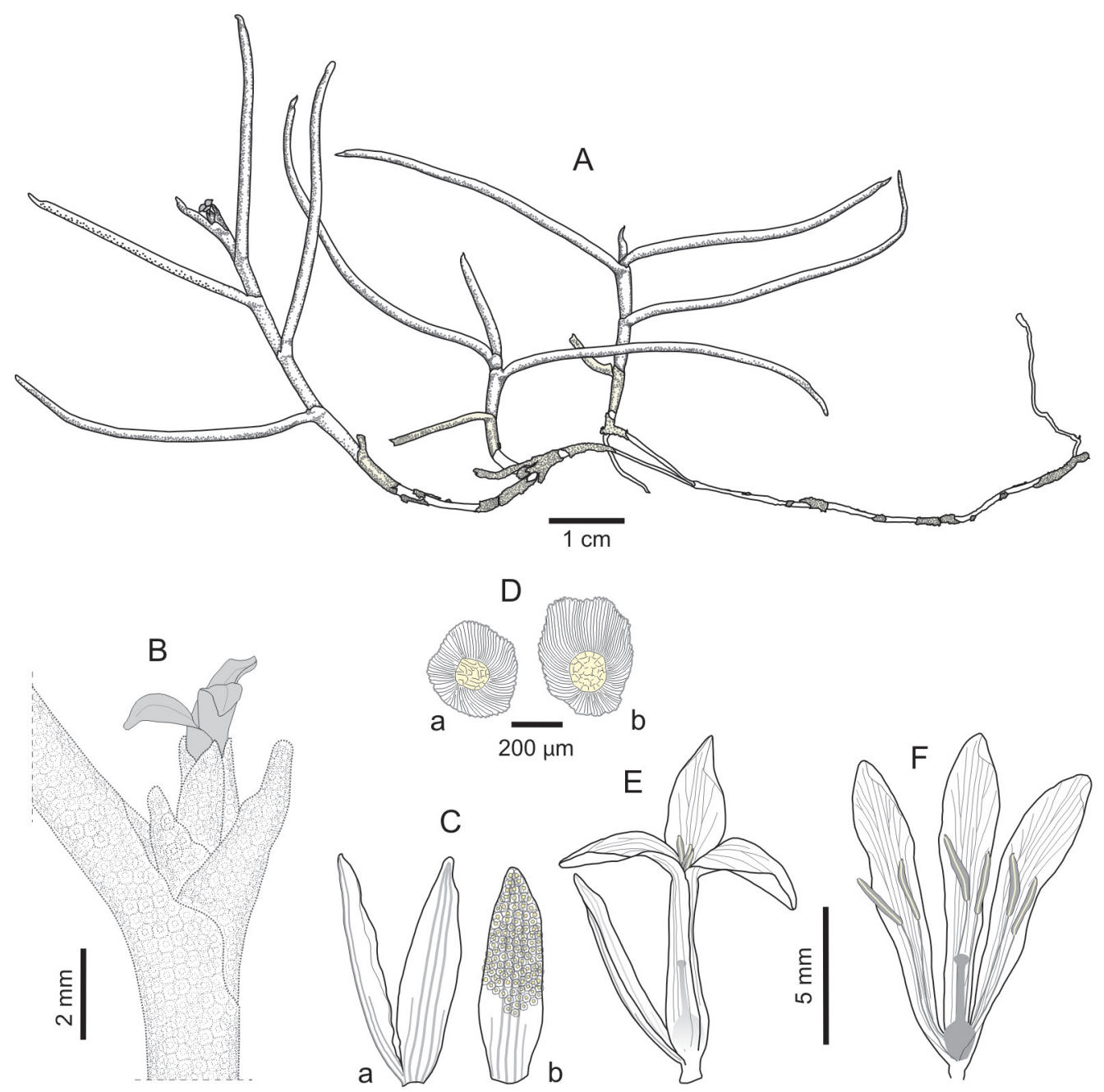

Fig. 2. Tillandsia mollis var. reptans. A: Aspecto de la planta. B: Inflorescencia. C: Sépalos, vista dorsal y lateral; a: desprovistos de escamas para hacer visible la nerviación; b: con escamas. D: Escamas; a: de los sépalos; b: de las hojas. E: Corola cerrada. F: Corola abierta.

mm, estilo 2 x 0,4 mm; estigma capitado. Cápsula sésil o subsésil, parda, conspicuamente mucronada en dispersión, 15-17 mm de longitud; exocarpo y endocarpo diferenciados. Semillas fusiformes con un apéndice plumoso en el ápice.

Distribución, hábito y fenología. Tillandsia mollis var. reptans sólo es conocida para las Sierras de Calilegua (Fig. 3), donde ocupa el nivel montano superior y la zona de pastizales de neblina, por encima de los $2500 \mathrm{~m}$ s.m. y comúnmente crece sobre paredones rocosos o acantilados (Fig. 1, A), conformando extensas colonias laxas de aspecto cespitoso (Fig. 1, B). La floración no es constante todos los años, o al menos no es muy visible, tiene lugar entre fines de invierno y primavera y los frutos están en dispersión desde inicios de verano.

Categoría en la Lista Roja de IUCN (2001). Es conocida sólo para la localidad tipo, Quebrada de Cortaderas, donde está muy poco extendida $\mathrm{y}$, hasta tanto se conozca el alcance de su distribución geográfica, la incluimos en la categoría Data Deficient (DD). 
Etimología. El nombre de la variedad hace referencia a su hábito dominante.

Obs. 1. En los ejemplares colectados en Calilegua es posible diferenciar dos tipos de tallos, por lo menos funcionalmente: tallos postrados radicantes y tallos erguidos foliados. Los tallos postrados, que se extienden adheridos al sustrato, están desprovistos de hojas, o con restos de vainas foliares en descomposición o totalmente desnudos. De algunos nudos crecen raíces simples, alargadas y fuertes con las que se adhieren al sustrato rocoso. Los tallos erguidos son cortos, de no más de $5 \mathrm{~cm}$ de alto, simples o escasamente ramificados y con muy pocas hojas (de 3 a 5). Las hojas son largas, divaricadas y levemente curvadas hacia arriba en el extremo. Los pétalos son castaño-rojizos.

Obs. 2. De los tres registros reportados de T. mollis, sólo fue posible consultar imágenes escaneadas de alta resolución del holotipo e isotipos (H. Hromandnik HR9088) depositados en WU y HAL, NY, W y WU, respectivamente. De estas observaciones y de la descripción original sumado al análisis de un reciente exhaustivo estudio sobre el subgénero Diaphoranthema (Donadío et al., 2015), se sostiene que T. mollis var. reptans difiere de la variedad típica por su hábito de crecimiento, parcialmente rastrero, por su porte notablemente menor y por el color de sus flores.

Material estudiado: ARGENTINA. Prov. Jujuy: Dpto. Valle Grande, Sierras de Calilegua, Quebrada de Cortaderas, 4-IX-2011 (LIL), Bulacio \& Ayarde 2031; Idem, 9-XII-2012, Bulacio \& Ayarde 2496 (LIL); Idem, 16-XI-2014, Bulacio \& Ayarde 2766 (LIL).

Sobre la base de las observaciones precedentes se elaboró la siguiente clave para diferenciar las dos variedades:

1. Plantas de crecimiento agregado, formando un cojín, tallos de 10-25 cm de largo por 3-4 mm de diámetro, entrenudos de hasta $8 \mathrm{~mm}$ de largo; hojas más de 10 por tallo, de hasta $40 \mathrm{~mm}$ de largo y de 2-3 mm de diámetro. Flores amarilloverdosas a amarillo-ocráceas.

T. mollis var. mollis

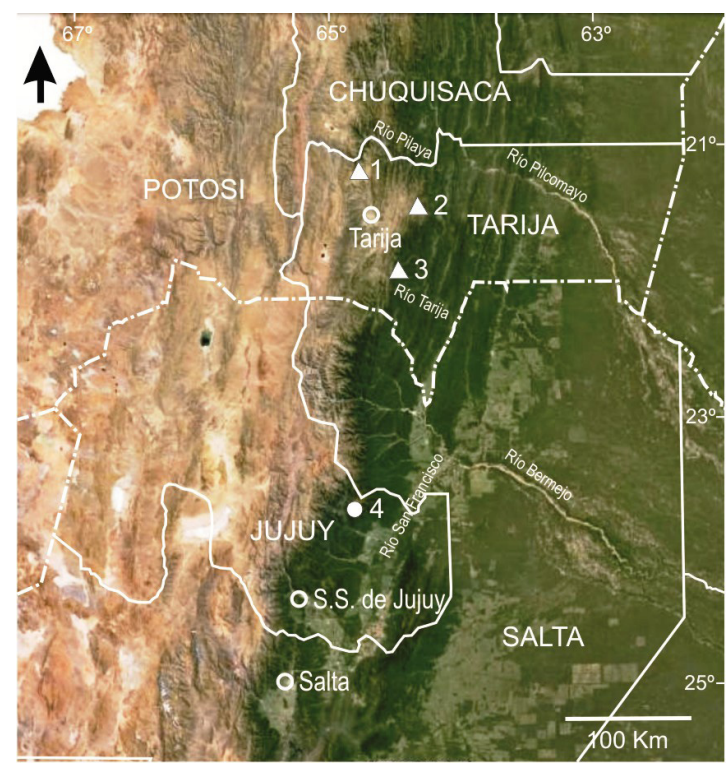

Fig. 3. Distribución geográfica de Tillandsia mollis. 1: Curso superior río Pilaya (Tarija, Bolivia), 2: Abra del Cóndor (Tarija, Bolivia), 3: Cerro Lorayoj (Tarija, Bolivia), 4: Sierras de Calilegua (Jujuy, Argentina). Triángulo $=T$. mollis var. mollis, Círculo $=T$. mollis var. reptans. Mapa elaborado sobre imagen tomada de Google Earth.

1'. Plantas de crecimiento rastrero, cespitosa, los tallos erguidos de hasta $5 \mathrm{~cm}$ de largo por 2 $\mathrm{mm}$ de diámetro, entrenudos de hasta $14 \mathrm{~mm}$ de largo; hojas de 3 a 5 por tallo, de hasta $70 \mathrm{~mm}$ de largo y de 1,1-1,8 mm de diámetro. Flores castaño-rojizas.

T. mollis var. reptans

\section{Agradecimientos}

A la Fundación Miguel Lillo por la provisión de los fondos para las campañas; a los curadores y técnicos de los herbarios LIL, CORD, MCNS, SI, LPB, BOLV, HSB, USZ y en especial al Dr. Chiarini (CORD) por el envío de la imagen escaneada del isosintipo de Tillandsia dependens var. perusneoides y de bibliografía de referencia y a revisores anónimos por la lectura crítica del texto.

\section{Biblografía}

BULACIO, E. \& H. AYARDE. 2009. Calilegua, algo más que un Parque Nacional. 9nas. Jornadas de 
Comunicaciones de la Fac. de Cs. Naturales, UNT y 2das. Jornadas Interinstitucionales FacultadFundación Miguel Lillo. Serie Monográfica y Didáctica 48: 18. Fac. Cs. Naturales e Inst. Miguel Lillo (UNT), Tucumán.

CASTELLANOS, A. 1945. Bromeliaceae Argentinae Novae vel criticae 4. Lilloa 11: 135-151.

CASTELlO, L. V. \& L. GALETTO. 2013. How many taxa can be recognized within the complex Tillandsia capillaris (Bromeliaceae, Tillandsioideae)? Analysis of the available classifications using a multivariate approach. PhytoKeys 23: 25-39.

DONADÍO, S., R. POZNER \& L. M. GIUSSANI. 2015. Phylogenetic relationships within Tillandsia subgenus Diaphoranthema (Bromeliaceae, Tillandsioideae) based on a comprehensive morphological dataset. $P l$. Syst. Evol. 301: 387-410.

IUCN, 2001. IUCN Red List Categories and Criteria, Version 3.1. Prepared by the IUCN Species Survival Commission. IUCN, Gland and Cambridge.

SMITH, L. B. \& R. J. DOWNS. 1977. Tillandsioideae (Bromeliaceae). Flora Neotropica 14 (2): 663-1492.
TILL, W. 1989. Die Untergattung Diaphoranthema (Beer) C. Koch von Tillandsia Linnaeus. 1. Das Tillandsia capillaris Aggregat. Die Bromelie 2/89: 31-34.

TILL, W. 1992a. Systematics and evolution of the tropicalsubtropical Tillandsia subgenus Diaphoranthema (Bromeliaceae). Selbyana 13: 88-94.

TILL, W. 1992b. Die Untergattung Diaphoranthema von Tillandsia. 4. Teil: Das Tillandsia recurvata Aggregat. Die Bromelie 1/1992: 15-20.

TILL, W. \& H. HROMADNIK. 1983. Tillandsia mollis (Bromeliaceae) eine neue Art aus Südbolivien. Pl. Syst. Evol. 142: 123-128.

TROPICOS.ORG. Missouri Botanical Garden. Acceso: 08 Jul $2015<$ http://www.tropicos.org/ Name/50008075>.

Recibido el 2 de diciembre de 2015, aceptado el 1 de abril de 2016. 